\title{
Proceeding
}

Supplementary Issue: Spring Conferences of Sports Science. Costa Blanca Sports Science Week, 26-28 April 2018. Calpe. Alicante, Spain

\section{Autonomous physical activity based program versus aerobic exercise based interventions to improve health and cardiovascular status in sedentary overweight adults: A review}

ROSA MARIA ALFONSO ROSA ${ }^{1} \mathrm{M}$, JUAN ANTONIO CORRAL PERNIA ${ }^{2}$, CAROLINA CASTAÑEDA VÁZQUEZ², JESUS DEL POZO CRUZ3

${ }^{1}$ Teaching area of Human Motricity and Sports Performance, University of Seville, Seville, Spain

${ }^{2}$ Research group HUM-507: Physical Education and Sport, University of Seville, Seville, Spain

${ }^{3}$ Department of Physical Education and Sports, University of Seville, Seville, Spain

\begin{abstract}
This review aims to identify improvements on health-related variables, produced by autonomous physical activity interventions and reducing sedentary behaviour, compared with improvements produced by interventions of aerobic exercise in sedentary and overweight / obesity. 17 design studies Randomized Controlled Trial (RCT) were finally included, with subjects $\geq 18$ years and a Body Mass Index (BMI) $\geq 25$. The results show similar results in both types of interventions, and the conclusion reached was that there is insufficient evidence of benefits of one type of intervention over another. Key words: SEDENTARY BEHAVIOUR, OBESITY, AUTONOMOUS PHYSICAL ACTIVITY, EXERCISE; HEALTH.
\end{abstract}

Cite this article as:

Alfonso Rosa, R.M., Corral Pernia, J.A., Castañeda Vázquez, C., \& del Pozo Cruz, J. (2018). Autonomous physical activity based program versus aerobic exercise based interventions to improve health and cardiovascular status in sedentary overweight adults: A review. Journal of Human Sport and Exercise, 13(2proc), S296-S308. doi:https://doi.org/10.14198/jhse.2018.13.Proc2.14

Corresponding author. Teaching area of Human Motricity and Sports Performance, University of Seville, Seville, Spain.

E-mail: roalrosa@us.es

Supplementary Issue: Spring Conferences of Sports Science. Costa Blanca Sports Science Week, 26-28 April 2018. Calpe. Alicante, Spain.

JOURNAL OF HUMAN SPORT \& EXERCISE ISSN 1988-5202

(c) Faculty of Education. University of Alicante

doi: 10.14198/jhse.2018.13.Proc2.14

S296 | 2018| Proc2 | VOLUME 13

C 2018 University of Alicante 


\section{INTRODUCTION}

Sedentary lifestyle is becoming one of the most serious problems that currently occur in westernized societies. The sedentary is caused by the shift towards less active life habits, due to the use and development of means of transport, the occupation of time in jobs with little physical requirements or new forms of leisure related to technology (Owen, 2012; Pate, O'Neill, \& Lobelo, 2008). Moreover, the lack of time due to long working hours and problems to reconcile family life with the practice of physical activity encourage the development of this public health problem.

Sedentary behavior is any waking behavior characterized by an energy expenditure $\leq 1.5$ metabolic equivalents (METs), while in a sitting, reclining or lying posture. In general, this means that any time a person is sitting or lying down, they are engaging in sedentary behaviour. Common sedentary behaviours include TV viewing, video game playing, computer use (collective termed "screen time"), driving automobiles, and reading (Mark S. Tremblay et al., 2017). But it is necessary to distinguish the concept of sedentary behavior from physical inactivity. Physical inactivity is defined as the lack of physical activity of moderate/vigorous intensity. According to the American College of Sport Medicine, a person is inactive when they perform less than 150 minutes a week of moderate physical activity or 75 minutes of vigorous physical activity (Garber et al., 2011).

On the other hand, there are many studies that relate sedentary behaviour with many diseases that cause a large number of deaths a year, such as diabetes mellitus type 2, coronary heart disease or cancer, so it is considered sedentary one of the factors of most serious risk at present (León-Latre et al., 2014; RodríguezHernández, Molina, Martínez-Santos, \& Cruz-Sánchez, 2011; Thorp, Owen, Neuhaus, \& Dunstan, 2011). In addition, sedentary behaviour has a high association with premature mortality, without the presence of the aforementioned diseases, and even independent of the level of physical activity practiced by the person (van der Ploeg, Chey, Korda, Banks, \& Bauman, 2012). Related to the prevalence of diseases caused largely by the sedentary lifestyle of the population, are the health costs that occur as a result of these and that would save large amounts of money and resources to states that promote public health strategies against sedentariness (Peeters, Mishra, Dobson, \& Brown, 2014).

Mention aside has the undoubted relationship that exists between sedentary lifestyle and overweight and obesity. These two considered global pandemics are inseparable in most cases. The sedentary lifestyle is positively correlated with obesity in both the adult population and children (Goldfield et al., 2013; MartínezGómez et al., 2010). Due to the characteristics of sedentary activities, the caloric expenditure produced is minimal, so the sedentary time will favor in excess a positive energy balance at the end of the day. Although it is not the objective of this systematic review, it is necessary to take into account the relationship between sedentary activities and the eating habits that exist during its realization (Goldfield et al., 2013). Although the existing relations between sedentary lifestyle and obesity are known, the possible causes are still unknown, although there is a diversity of theories that seem to be combined (Mark Stephen Tremblay, Colley, Saunders, Healy, \& Owen, 2010).

For all the above, the concern from the scientific field for sedentary lifestyle and its consequences has grown in recent years, in which different interventions related to the decrease in sedentary time and increased physical activity have emerged (Dutheil et al., 2013; Foster, Gore, \& West, n.d.; Steeves, Bassett, Fitzhugh, Raynor, \& Thompson, 2012). These interventions are very diverse and range from reducing the hours of watching television or increasing the number of steps, to supervised aerobic trend training. Hence the problem of research addressed in this systematic review whose main objective is to establish the results in 
different variables related to the health of interventions based on unsupervised physical activity in sedentary and overweight / obese people and compare them with the results obtained in supervised aerobic trend exercise interventions in the same population.

\section{METHODS}

The study was undertaken in accordance with the Preferred Reporting Items for Systematic Reviews and Meta-Analyses (PRISMA) statement (Liberati et al., 2009).

\section{Data sources}

Literature search was conducted (September 2015) in PubMed, Web of Science (WoS) and Scopus online data bases. Limit on the date of publication was imposed to 2010 until 2015. The search strategy was performance following the words: "adult", "aged", "obesity", "overweight", "waist circumference", "waist hip ratio", "body size", "body constitution", "weight", "BMl", "quality of life", "hrqol", "sedentary time", "sitting time", "screen time", "steps", "counts", "acceleromet", "objetive measured", "pedometers", "lifestyle", "life change events", "sedentary lifestyle", "sedentary behavior", "lifestyle change", "television", "tv", "reduce sitting time", "reduce screen time", "activities of daily", "leisure activities", "aerobic training", "endurance training", "randomized controlled trial". Also, the reference lists of included articles were examined to detect studies potentially eligible for inclusion. Non-English studies were not explored.

\section{Eligibility criteria}

Studies were included in the review if they met the following inclusion criteria: (i) included human participants aged $\geq 18$ years; (ii) people considered sedentary and overweight/obese; (iii) interventions on sedentary lifestyle carried out autonomously or that carried out supervised aerobic training; (iv) written in English; and (v) randomized controlled trial (RCT). Studies were not included in the review if they met the following exclusion criteria: (i) interventions aimed at weight loss in which some obligatory change in the diets will be made; (ii) pregnant women.

Two independent reviewers carried out the screening and review, with a third reviewer sought in case of disagreement. Articles were first screened for eligibility based on title and abstract. The full text was then reviewed, and after confirming eligibility to be included, data were extracted.

\section{Risk of bias}

The PEDro scale was used to assess the risk of bias in the selected articles. This is a scale that rates the methodological quality of RCTs that evaluate physical therapist interventions. This scale was chosen because of its special design and capacity to provide a global overview of the external and internal validity of the studies (Maher, Sherrington, Herbert, Moseley, \& Elkins, 2003). Each article was graded by one of the authors, and this grading was supervised by another author with experience in this task. Table 1 shows the consensus results for each article.

\section{Data Extraction and the Main Measurement Examined}

Data were extracted from all articles that met selection criteria and deemed to be appropriated for detailed review by two authors, and differences were discussed. Information extracted was as follow: characteristics of the sample, duration and characteristics of the intervention protocol, gender and age of the participants. Also, data related to different parameters of health, variables of vital importance in sedentary and overweight/obese people (\% body fat, body mass index, weight, glycemic control, blood pressure, circumference waist, lipid profile were extracted. 
Table 1.

Risk of bias in studies with unsupervised interventions

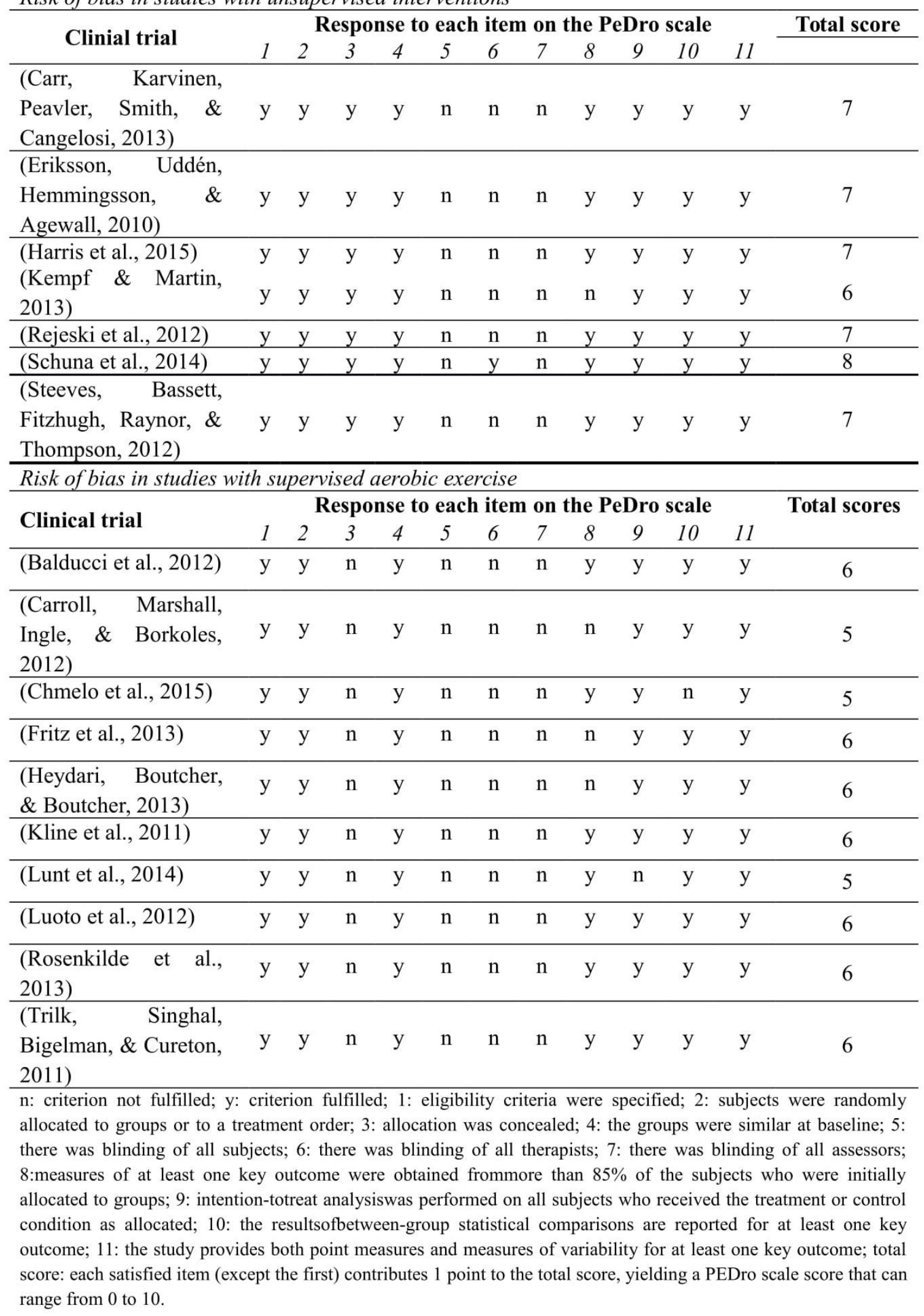




\section{RESULTS}

Figure 1 depicts the process that was followed in this systematic review. In total, 315 articles were found in the electronic search database. After removing the duplicates, 216 references were reviewed. Of these, 199 were excluded because a review of their summaries revealed that the study clearly did not meet the inclusion criteria. Finally, 17 articles were included in our systematic review. Thus, in total 17 full-text articles were finally included in the review (a summary of the most relevant study details of these studies are included in Table 2).

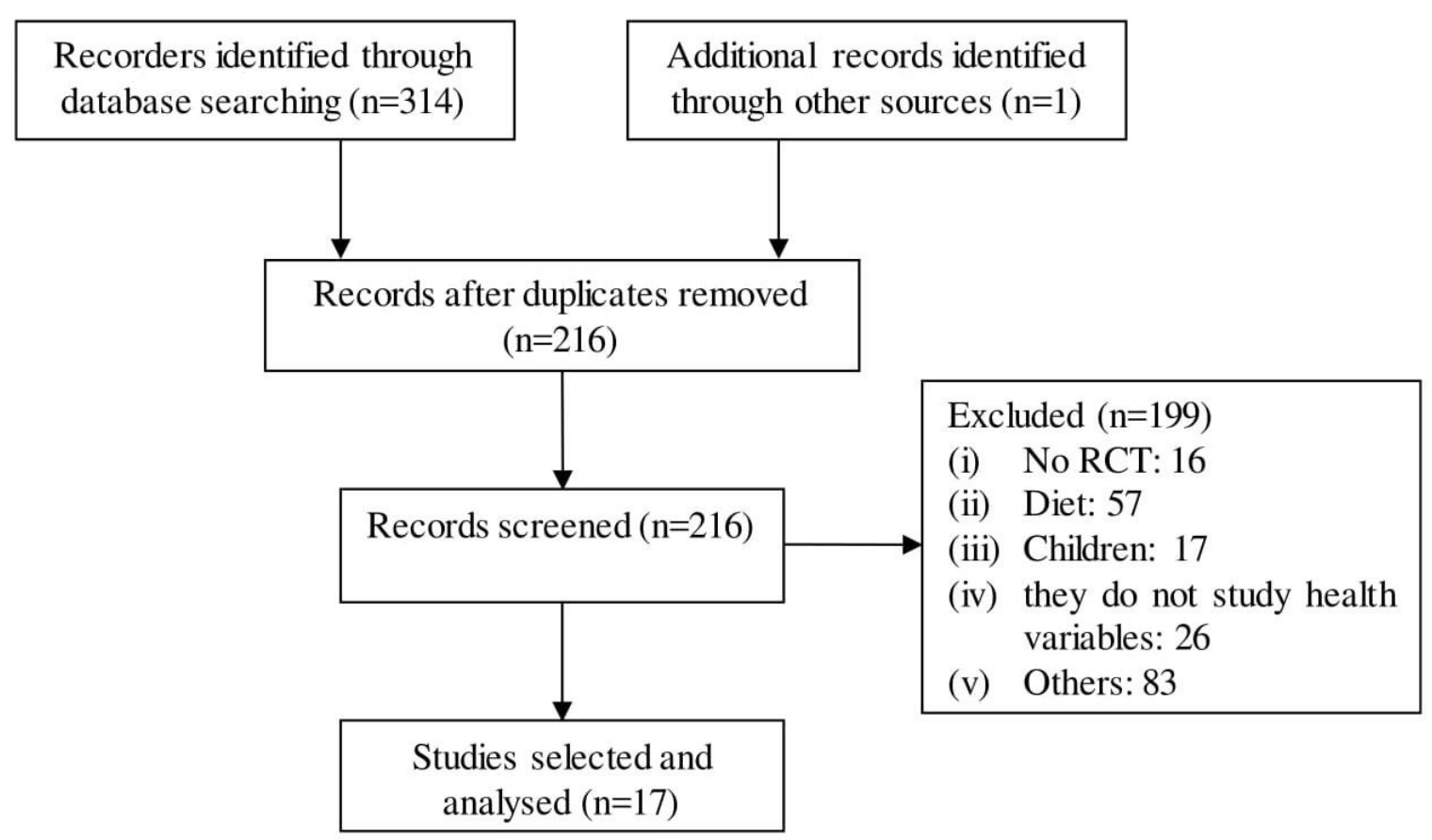

Figure 1. Flow diagram

\section{Unsupervised interventions}

For this type of interventions only 7 trials were included (Table 3). For the variable $\%$ body fat, changes have been collected in some of the interventions but only Harris et al., (2015) significant differences were obtained $(p=0.1)$.

Regarding the BMI only change was found by Kempf \& Martin (2013) $(-0.4 \pm 1.6$ with $p=0.001)$. The results referring to the weight show statistically significant changes in two studies (Kempf \& Martin, 2013; Rejeski et al., 2012). In addition, there is heterogeneity in the changes, since depending on the intervention, weight losses are obtained from $0.5 \mathrm{~kg}$ to $6.87 \mathrm{~kg}$.

Based on blood pressure, both systolic and diastolic blood pressure were chosen for the analysis. For systolic blood pressure significant changes were obtained by Eriksson, Uddén, Hemmingsson, \& Agewall (2010) [-7 $\mathrm{mmHg} p=0.0002$ ] and Carr, et al. (2013) $-(4.3 \mathrm{mmHg} p=0.002)$, while for diastolic blood pressure the change only it is significant by Ericksson et al., (2010) $(-2 \mathrm{mmHg}, \mathrm{p}=0.0003)$. For the WHR variable, statistically significant changes were found, -2.5 and $-1.6 \mathrm{~cm} p<0.05$ in the two groups of the Steeves study (Steeves et al., 2012); -2.4 p=0. 0003 in Ericksson study (2010) and -1 p=0.06 in the study of Carr, et al., (2013). 
Table 2.

Characteristics of the studies with unsupervised interventions

\begin{tabular}{|c|c|c|c|c|c|}
\hline Author (Study) & Sample & Duration & Gender & Age & Intervention \\
\hline $\begin{array}{l}\text { (Carr, Karvinen, } \\
\text { Peavler, Smith, \& } \\
\text { Cangelosi, 2013) }\end{array}$ & $\begin{array}{l}\text { CG:17 } \\
\text { EG:23 }\end{array}$ & 12 weeks & W-M & $35-55$ & $\begin{array}{l}\text { Reduce sedentary time, portable } \\
\text { pedals and motivating messages. }\end{array}$ \\
\hline $\begin{array}{l}\text { (Eriksson, Uddén, } \\
\text { Hemmingsson, } \quad \text { \& } \\
\text { Agewall, 2010) }\end{array}$ & EG: 50 & 6 months & W & $30-60$ & $\begin{array}{l}\text { Increase time walking and cycling, } \\
\text { especially on the way to work. }\end{array}$ \\
\hline (Harris et al., 2015) & $\begin{array}{l}\text { CG: } 148 \\
\text { EG: } 150 \\
\end{array}$ & 12 weeks & W-M & $60-75$ & $\begin{array}{l}\text { Sessions to incite increase number } \\
\text { of steps. }\end{array}$ \\
\hline $\begin{array}{l}\text { (Kempf \& Martin, } \\
\text { 2013) }\end{array}$ & $\begin{array}{l}\text { CG: } 100 \\
\text { EG: } 120\end{array}$ & 12 weeks & W-M & $50-70$ & $\begin{array}{l}\text { Autonomous exercise with Wii } \\
\text { (30min / day). }\end{array}$ \\
\hline (Rejeski et al., 2012) & $\begin{array}{l}\text { CG:2506 } \\
\text { EG:2510 }\end{array}$ & $\begin{array}{l}1 \text { year; } \\
\text { follow up } \\
\text { until the } \\
4 \text { th year }\end{array}$ & W-M & $50-70$ & Awareness talks, debates. \\
\hline (Schuna et al., 2014) & $\begin{array}{l}\text { CG: } 20 \\
\text { EG: } 21\end{array}$ & 12 weeks & W & $30-50$ & Desk with ergometer (90min/day) \\
\hline $\begin{array}{l}\text { (Steeves, Bassett, } \\
\text { Fitzhugh, Raynor, \& } \\
\text { Thompson, 2012) }\end{array}$ & $\begin{array}{l}\text { CG: } 29 \\
\text { EG: } 29\end{array}$ & 6 months & W-M & $40-60$ & $\begin{array}{l}\text { G1: } 30 \text { min walk, } 5 \text { days a week } \\
\text { G2: Walk in all the ads of a } \\
\text { television program of at least } 90 \\
\text { min. } 5 \text { times a week. }\end{array}$ \\
\hline \multicolumn{6}{|c|}{ Characteristics of the studies with supervised interventions } \\
\hline (Balducci et al., 2012) & $\begin{array}{l}\text { CG: } 39 \\
\text { EG: } 34\end{array}$ & 12 months & W-M & $50-70$ & $\begin{array}{l}2 \text { sessions } 75 \text { min each (aerobic } \\
\text { and resistance training) }\end{array}$ \\
\hline $\begin{array}{l}\text { (Carroll, } \\
\text { Ingle, \& } \begin{array}{l}\text { Marshall, } \\
\text { 2012) }\end{array}\end{array}$ & $\begin{array}{l}\text { CG: } 31 \\
\text { EG:30 }\end{array}$ & 12 weeks & W & $35-45$ & $\begin{array}{l}\text { Intervention on lifestyle and } \\
\text { diverse training sessions. }\end{array}$ \\
\hline (Chmelo et al., 2015) & $\begin{array}{l}\text { AG: } 40 \\
\text { RTG: } 55\end{array}$ & 5 months & W-M & $65-79$ & $\begin{array}{l}\text { Strength training ( } 3 \text { days a week }= \\
\text { vs aerobic training ( } 4 \text { days a } \\
\text { week). }\end{array}$ \\
\hline (Fritz et al., 2013) & $\begin{array}{c}\mathrm{G}_{1}: 128(75 \\
\mathrm{CG} / 53 \mathrm{EG}) \\
\mathrm{G}_{2}: 35(21 \mathrm{CG} / 14 \\
\mathrm{EG}) \\
\mathrm{G}_{3}: 52(30 \mathrm{CG} / 20 \\
\mathrm{EG})\end{array}$ & 4 months & W-M & $45-69$ & Nordic walking (5 h a week). \\
\hline $\begin{array}{l}\text { (Heydari, Boutcher, \& } \\
\text { Boutcher, 2013) }\end{array}$ & $\begin{array}{l}\text { CG: } 17 \\
\text { EG: } 17\end{array}$ & 12 weeks & M & $18-35$ & $\begin{array}{l}\text { HIIT, } 3 \text { times a week. In cycle } \\
\text { ergometer. } 80-90 \% \text { Fcmax. for } 20 \\
\text { min, } 8 \text { s of interval and } 12 \text { s rest }\end{array}$ \\
\hline (Kline et al., 2011) & $\begin{array}{c}\text { CG } \\
\text { (stretching): } \\
16 \\
\text { EG: } 27 \\
\end{array}$ & 12 weeks & W-M & $40-50$ & $\begin{array}{l}4 \text { times per week. } 150 \text { min aerobic } \\
\text { a week and } 2 \text { days resistance } \\
\text { training } 2 \times 10-12 \text { ( } 8 \text { exercises) }\end{array}$ \\
\hline (Lunt et al., 2014) & $\begin{array}{c}\text { AITG: } 16 \\
\text { HIITG: } 16 \\
\text { WG (control): } \\
17\end{array}$ & 12 weeks & W-M & $40-55$ & 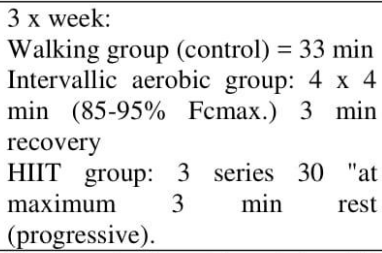 \\
\hline (Luoto et al., 2012) & $\begin{array}{l}\text { CG: } 77 \\
\text { EG: } 74\end{array}$ & 6 months & W & $40-63$ & $\begin{array}{l}\text { Unsupervised aerobic training ( } 50 \\
\text { min / } 4 \text { times per week) } 64-80 \% \\
\text { Fcmax. Mixed activities. }\end{array}$ \\
\hline $\begin{array}{l}\text { (Rosenkilde et al., } \\
\text { 2013) }\end{array}$ & $\begin{array}{l}\text { CG: } 18 \\
\text { MG: } 21 \\
\text { IG: } 22\end{array}$ & 12 weeks & M & $20-40$ & $\begin{array}{l}\text { Aerobic training: moderate group } \\
(+300 \text { kcal per day }) \\
\text { Intense group }(+600 \text { kcal per day). }\end{array}$ \\
\hline $\begin{array}{l}\text { (Trilk, Singhal, } \\
\text { Bigelman, \& Cureton, } \\
\text { 2011) }\end{array}$ & $\begin{array}{l}\text { CG: } 14 \\
\text { EG: } 14\end{array}$ & 4 weeks & W & $25-35$ & $\begin{array}{l}\text { Sprint interval training. } 3 \text { times per } \\
\text { week. } 4-7 \text { series of } 30 \mathrm{~s} \text { on cycle } \\
\text { ergometer. }\end{array}$ \\
\hline
\end{tabular}

CG: Control Group; EG: Experimental Group; W: Woman; M: Man; $\mathrm{G}_{1}$ : normal glucose tolerance; $\mathrm{G}_{2}$ : impaired glucose tolerance; $\mathrm{G}_{3}$ : diabetes mellitus type 2; AITG: aerobic interval training group; MG: Moderate Group; IG: Intensity Group 
Table 3.

Outcome measure in studies with unsupervised interventions

\begin{tabular}{|c|c|c|c|}
\hline Study & Variables & Pre & Post \\
\hline \multirow{8}{*}{$\begin{array}{l}\text { (Carr, Karvinen, } \\
\text { Peavler, Smith, \& } \\
\text { Cangelosi, 2013) }\end{array}$} & BMI & $31.7(4.9)$ & $31.9(5.0)-0.1 \mathrm{sc}$ \\
\hline & Weight $(\mathrm{kg})$ & $88(15.8)$ & $88.2(15.6)+0.2 \mathrm{sc}$ \\
\hline & Blood pressure & SP: $120.0(13.8)$ & SP: $115.7(10.8)-4.3 p=0.02$ \\
\hline & $(\mathrm{mmHg})$ & DP: $78.2(10.3)$ & DP: $75.4(7.4)-2.8 p=0.11$ \\
\hline & Waist & $92.6(11.2)$ & $91.6(11.3)-1.0 \mathrm{p}=0.06$ \\
\hline & & Total C: 191.4 (26.3) & Total C: $189.7(27)-.7^{*}$ \\
\hline & Lipid profile & HDL: 45.7 (17.6) & HDL: 43.7 (16.4) -2.1* \\
\hline & & LDL: 119.4 (23.2) & LDL: 116.7 (29.4) -3.7* \\
\hline \multirow{4}{*}{$\begin{array}{l}\text { (Eriksson, Uddén, } \\
\text { Hemmingsson, \& } \\
\text { Agewall, 2010) }\end{array}$} & Weight $(\mathrm{kg})$ & $84.4(10)$ & $83.9(11.2)-0,5 \mathrm{p}=0.08$ \\
\hline & Blood pressure & SP: $127(15)$ & SP: $120(15)-7 \mathrm{p}=0.0002$ \\
\hline & $(\mathrm{mmHg})$ & DP: $80(8)$ & DP: $78(8)-2 p=0.0003$ \\
\hline & $\begin{array}{l}\text { Waist } \\
\text { circumference }\end{array}$ & $103.2(7.8)$ & $100.8(8.4)-2.4 p=0.0003$ \\
\hline \multirow[t]{2}{*}{ (Harris et al., 2015) } & $\%$ body fat & $24.5(8.5)$ & $23.9(8.6)-0.6 \mathrm{p}=0.1$ \\
\hline & BMI & $27.7(4.1)$ & $27.6(4.1)^{*}$ \\
\hline \multirow{8}{*}{$\begin{array}{l}\text { (Kempf \& Martin, } \\
\text { 2013) }\end{array}$} & BMI & $34.1(6.5)$ & $33.5(6.5)-0.4 \mathrm{p}=0.0001$ \\
\hline & Weight $(\mathrm{kg})$ & $98(19)$ & $96(19)-1.2(4.7) p=0.0001$ \\
\hline & $\begin{array}{l}\text { Glycemic } \\
\text { control }\end{array}$ & $\begin{array}{c}\text { G. postprandial } \\
\text { (mg/dl): } 136(39) \\
\text { HbAlc (\%): } 7.1(1.3)\end{array}$ & $\begin{array}{l}\text { G. postprandial: } 127 \text { (37) } \\
-8(35) \mathrm{p}=0.041 \\
\mathrm{HbA} 1 \mathrm{c}(\%): 6.8(1.0)-0.3 \% \\
\mathrm{p}=0.0002\end{array}$ \\
\hline & Blood pressure & SP: $134(15)$ & SP: $132(13)-2(15) *$ \\
\hline & (mmHg) & DP: $80(8)$ & DP: $79(8)-0.6(10)^{*}$ \\
\hline & & Total C: 207 (43) & Total C: $204(44)-3(23)$ * \\
\hline & Lipid profile & HDL: 50 (14) & HDL: $51(14) *$ \\
\hline & & LDL: 125 (35) & LDL: $125(36)^{*}$ \\
\hline (Rejeski et al., 2012) & Weight $(\mathrm{kg})$ & $100.5(19.7)$ & $93.6(19.6)-6,87 p<0.001$ \\
\hline \multirow[t]{3}{*}{ (Schuna et al., 2014) } & $\%$ body fat & $46.1(1.6)$ & $44.9(1.4)-1,2^{*}$ \\
\hline & BMI & $36.8(2.4)$ & $36.6(2.3)-0,2^{*}$ \\
\hline & Weight $(\mathrm{kg})$ & $99.1(7.3)$ & $98.6(7.2)-0,5^{*}$ \\
\hline \multirow{8}{*}{$\begin{array}{l}\text { (Steeves, Bassett, } \\
\text { Fitzhugh, Raynor, \& } \\
\text { Thompson, 2012) }\end{array}$} & $0 /$ hodv fat & $\mathrm{G}_{1}: 42.4(6.9)$ & $\mathrm{G}_{1}: 41.8(7.2)-0.6^{*}$ \\
\hline & $\% 00$ & $\mathrm{G}_{2}: 41.3(6.3)$ & $\mathrm{G}_{2}: 40.4(6.8)-0.9 *$ \\
\hline & BMI & $\mathrm{G}^{\mathrm{l}}: 34.2(5.5)$ & $\mathrm{G}_{1: 34.0}(6.2)-0.2^{*}$ \\
\hline & BMII & $\mathrm{G}_{2:} 32.8(3.9)$ & $\mathrm{G}_{2:} 32.7(4.0)-0.1^{*}$ \\
\hline & Weight $(\mathrm{k} \alpha)$ & G1: $94.3(17.5)$ & G1: $93.6(17.7)-0.7^{*}$ \\
\hline & Welgnt (kg) & G2: $93.3(14.9)$ & G2: 92.9 (14.9) - $0.4^{*}$ \\
\hline & Waist & G1: 105.4 (13.6) & G1:102.9 (12.4) $-2,5 \mathrm{p}<0.05$ \\
\hline & circumference & G2: $102.3(8.1)$ & G2: $107(8.0)-1,6 \mathrm{p}<0.05$ \\
\hline
\end{tabular}




\section{Table 4.}

Outcome measure in studies with supervised interventions

\begin{tabular}{|c|c|c|c|}
\hline Study & Variables & Pre & Post \\
\hline \multirow{6}{*}{ (Balducci et al., 2012) } & $\mathrm{BMI}$ & $31.8(5.3)$ & $30.7(4.9)-1,1 p<0.001$ \\
\hline & $\begin{array}{l}\text { Glycemic } \\
\text { control }\end{array}$ & HbA1c (\%): 8.11 (1.82) & $\begin{array}{c}\mathrm{HbA} 1 \mathrm{c}(\%): 7.08(1.14)-1,03 \\
\mathrm{p}<0.001\end{array}$ \\
\hline & Blood pressure & SP: $143(20)$ & SP: $136(14)-7 p=0.001$ \\
\hline & $(\mathrm{mmHg})$ & DP: $82(11)$ & DP: $79(8)-3 p=0.012$ \\
\hline & $\begin{array}{l}\text { Waist } \\
\text { circumference }\end{array}$ & $107.4(13.6)$ & $103.6(11.9)-3,8 p<0.001$ \\
\hline & Lipid profile & $\begin{array}{l}\text { Total C: } 4.92(0.85) \\
\text { HDL: } 1.13(0.28) \\
\text { LDL: } 3.26(0.99)\end{array}$ & $\begin{array}{c}\text { Total C: } 4.64(1.19)-0,28 \\
p=0.2 \\
\text { HDL: } 1.20(0.33)+0,7 p=0.25 \\
\text { LDL: } 2.85(0.84)-0,41 p=0.04\end{array}$ \\
\hline \multirow{2}{*}{$\begin{array}{l}\text { (Carroll, Marshall, Ingle, } \\
\text { \& Borkoles, 2012) }\end{array}$} & BMI & $39.0(6.4)$ & $38.3(7.0)-0,7 p=0.003$ \\
\hline & Weight $(\mathrm{kg})$ & $106.6(17.8)$ & $104.6(19.2)-2 p=0.004$ \\
\hline \multirow{5}{*}{ (Chmelo et al., 2015) } & $\%$ body fat & $\begin{array}{l}\text { AG: } 44.2(5.3) \\
\text { RT: } 38.4 \pm 6.4\end{array}$ & $\begin{array}{l}\text { AG: } 43.7(5.3)-0.5 \\
\text { RT: } 37.8(6.6)-0.6\end{array}$ \\
\hline & & AG: $34.1(3.1)$ & AG: $33.6(3.5)-0.5$ \\
\hline & BMI & RT: $38.4(6.4)$ & RT: 30.6 (2.7) -7,8 p<0.05 \\
\hline & Waist & AG: $104.3(11.1)$ & $A G: 102.5(11.7)-1,8 p<0,05$ \\
\hline & circumference & RT: $96.2(9.4)$ & RT: $94.7(8.2)-1.5 p<0.05$ \\
\hline \multirow{10}{*}{ (Fritz et al., 2013) } & $\mathrm{BMI}$ & $\begin{array}{l}\mathrm{G}_{1}: 29.6(3.8) \\
\mathrm{G}_{2}: 32.0(5.2) \\
\mathrm{G}_{3}: 31.7(5.2)\end{array}$ & $\begin{array}{c}\mathrm{G}_{1:}-0.8(1.4) p<0.0001 \\
\text { G2: }-0.1(0.9)^{*} \\
\text { DMT2: }-0.4(0.8)\end{array}$ \\
\hline & Weight (kg) & $\begin{array}{c}\mathrm{G}_{1}: 85.2(13.7)-2(3.8) \\
p<0.001 \\
G 2: 92.5(14.7)-0.5(2.2) \\
G 3: 91.9(13.1)-1.0(2.1) \\
p<0.05\end{array}$ & $\begin{array}{c}G_{1}:-2.0(3.8) p<0.001 \\
G 2:-0.5(2.2) * \\
\text { G3: }-1.0(2.1) p<0.05\end{array}$ \\
\hline & $\begin{array}{l}\text { Glycemic } \\
\text { control }\end{array}$ & $\begin{array}{l}\mathrm{G}_{1}: \mathrm{HbA} 1 \mathrm{c}(\%) 5.7(0.3) \\
\text { G2: G. Post 2h (mmol) } \\
\text { 10.1(1) } \\
\text { G3:Hb1Ac\% 6.9(0.9) }\end{array}$ & $\begin{array}{c}G_{1}:{ }^{*} \\
\text { G2: } H_{b A 1 c}{ }^{*} G . \text { Post } 2 h: \\
0.7(1.0) p<0.05 \\
\text { G3: } \begin{array}{c}H b 1 A c \%-0.3(0.6) \\
p<0.05\end{array}\end{array}$ \\
\hline & & $\begin{array}{l}\text { G1: SP: } 138(12.5) \\
\text { DP: } 85(7.9)\end{array}$ & $\begin{array}{c}\text { G1: SP: +2.0(12.2) } \\
\text { G2: SP: }-0.7(20.0): D P:+\end{array}$ \\
\hline & Blood pressure & G2: SP: $141(14.0)$ & $1.8(11.2)$ \\
\hline & $(\mathrm{mmHg})$ & DP: 84(7.8) & G3: SP: +0.3(15.5) * \\
\hline & & $\begin{array}{l}\text { G3: SP: } 143(13.2) \\
\text { DP: } 85(7.6)\end{array}$ & $D P:-1.3(11.9) *$ \\
\hline & & $\mathrm{G}_{1}: 99.4(11.3)$ & $G_{1}:-4.9(4.4) p<0.001$ \\
\hline & Vircumforonco & G2: $103.7(10.5)$ & G2: $-2.4(3.0) p<0.05$ \\
\hline & circuminerence & G3: $105.6(10.3)$ & G3:-1.3(2.7) $p<0.05$ \\
\hline
\end{tabular}




\begin{tabular}{|c|c|c|c|}
\hline & Lipid profile & $\begin{array}{l}\text { G1: Total }(\mathrm{mmol}) \\
5.4(0.9) \\
\text { LDL: } 3.2(0.8) \\
\text { HDL: } 1.6(0.4) \\
\text { G2: Total }(\mathrm{mmol}) \\
5.5(0.9) \\
\text { LDL: } 3.2(0.8) \\
\text { HDL: } 1.5(0.4) \\
\text { G3: Total (mmol): } 4.8(0.9) \\
\text { LDL: } 2.7(0.8) \\
\text { HDL: } 1.4(0.3)\end{array}$ & $\begin{array}{c}\text { Total (mmol): }-0.04(0.6) \\
\text { LDL: }-0.04(0.6) \\
\text { HDL: }-0.02(0.2)^{*} \\
\text { Total (mmol): } 0.04(0.5) \\
\text { LDL: } 0.03(0.1) \\
\text { HDL: } 0.04(0.5)^{*} \\
\text { G3: Total (mmol): }-0.1(0.5) \\
\text { LDL: }-0.005(0.2) \\
\text { HDL: }-0.04(0.4)^{*}\end{array}$ \\
\hline \multirow{4}{*}{$\begin{array}{l}\text { (Heydari, Boutcher, \& } \\
\text { Boutcher, 2013) }\end{array}$} & BMI & $28.4(0.6)$ & $27.9(0.6)-0,5 p<0.05$ \\
\hline & Weight (kg) & $89.2(2.9)$ & $87.6(2.9)-1,6 p<0.05$ \\
\hline & $\begin{array}{l}\text { Blood pressure } \\
(\mathrm{mmHg})\end{array}$ & $\begin{array}{l}\text { SP: } 120(2.4) \\
\text { DP: } 64(1.8)\end{array}$ & $\begin{array}{l}\text { SP: } 115(2.5)-5 p<0.05 \\
\text { DP: } 58(1.8)-6 p<0.05\end{array}$ \\
\hline & $\begin{array}{l}\text { Waist } \\
\text { circumference }\end{array}$ & $93.5(1.6)$ & $89.8(1.7)-3,7 p<0.05$ \\
\hline \multirow{4}{*}{ (Kline et al., 2011) } & $\%$ body fat & 42.1(1.9) & $41.0(1.9)-1.1 p<0.05$ \\
\hline & $\mathrm{BMI}$ & $35.5(1.2)$ & - \\
\hline & Weight (kg) & $105.6(3.0)$ & $104.7(3.1)-0.9^{*}$ \\
\hline & $\begin{array}{l}\text { Waist } \\
\text { circumference }\end{array}$ & $110.8(2.3)$ & $110.2(2.3)$ \\
\hline (Luoto et al., 2012) & $\%$ body fat & $27.2(8.8)$ & $26.1(8.6)-0.9 p=0.219$ \\
\hline \multirow{4}{*}{ (Rosenkilde et al., 2013) } & $\%$ body fat & $\begin{array}{l}\text { MG: } 30.0(4.6) \\
\text { IG: } 27.4(4.2)\end{array}$ & $\begin{array}{l}\text { MG: } 25.8(5.1)-4.2 p<0.001 \\
\text { IG: } 23.7(3.7)-3.7 \% p<0.001\end{array}$ \\
\hline & $\mathrm{BMI}$ & $\begin{array}{l}\text { MG: } 28.6(1.8) \\
\text { IG: } 27.6(1.4)\end{array}$ & $\begin{array}{c}\text { MG: } 27.5(2.0)-1.1 p<0.001 \\
\text { IG: } 26.9(1.2)-0.7 p=0.01\end{array}$ \\
\hline & Weight (kg) & $\begin{array}{l}\text { MG: } 93.2(8.1) \\
\text { IG: } 91.3(7.2)\end{array}$ & $\begin{array}{c}\text { MG: } 89.7(8.6)-3.5 p<0.001 \\
\text { IG: } 88.8(6.7)-2.1 p=0.01\end{array}$ \\
\hline & $\begin{array}{l}\text { Glycemic } \\
\text { control }\end{array}$ & $\begin{array}{l}\text { MG: } 5.4(0.2) \\
\text { IG: } 5.6(0.3)\end{array}$ & $\begin{array}{c}\text { MG: } 5.3(0.2)-0.1 p=0.02 \\
\text { IG: } 5.5(0.3)-0,1^{*}\end{array}$ \\
\hline
\end{tabular}

*No significant changes; BMl: Body Mass Index; SP: Systolic Pressure; DP: Diastolic Pressure; Total C: Total Cholesterol; HDL: High Density Lipoprotein; LDL: Low Density Lipoprotein; AG: Aerobic Group; RG: Resistance training group; $\mathrm{G}_{1}$ : normal glucose tolerance; $\mathrm{G}_{2}$ : impaired glucose tolerance; $\mathrm{G}_{3}$ : diabetes mellitus type 2; MG: Moderate Group; IG: Intensity Group

\section{Supervised interventions}

The interventions based on physical exercise finally reported 10 studies to this systematic review (Table 4). The same variables as in the previous section were studied in order to make a comparison of the results. In the $\%$ body fat variable, it was measured in 6 of the 10 articles and there are only significant changes by Kline et al., (2011) $-1.1 p<0.05$, Lunt et al., (2014) with $-0.9 \%$ p $<0.001$ in the total of the different groups and Rosenkilde et al., (2013) $-4.2 \%$ and $-3.7 \%$ with $p<0.001$ in the groups moderate intensity training and high respectively. The BMI was analyzed in 9 of the 10 studies, reporting significant changes Carroll et al., (2012)- 
$0.7 p<0.003$, Rosenkilde et al., (2013) -1.1 and $-0.7 p<0.001$, Fritz et al., (2013) $-0.8 \pm 1.4 p<0.0001$ only in the group with normal glucose tolerance. Chmelo et al., (2015) obtained change only in the group of resistance training $(-7.8 p<0.05)$. The weight was examined in 6 studies, obtaining 4 of them statistically significant changes: Carrol et al., (2012) $-2 \mathrm{~kg} p<0.004$, Rosenkilde et al., (2013) $-3.5 \mathrm{~kg} p<0.001$ in the moderate group and $-2.1 p<0.01$ in the group of high intensity, Fritz et al., (2013)-2.0kg, $p<0.001$ in the group of normal glucose tolerance and $-1.0 \mathrm{~kg} \mathrm{p}<0.05$ in the group of diabetics type 2 and Heydari et al., (2013) with $-1,6 \mathrm{~kg} \mathrm{p}<0.05$.

Regarding blood pressure, 3 study obtained significant changes: Lunt et al., (2014) found statistically significant differences in systolic blood pressure $(-4.8 \mathrm{mmHg}, \mathrm{p}=0.001)$ and diastolic blood pressure $(-2.6$ $\mathrm{mmHg} p=0.004)$. Finally, Balducci et al. (2012) obtained improvements of $-7 \mathrm{mmHg} p=0.001$ in the systolic pressure and $-3 p=0.012$ in the diastolic pressure.

The waist circumference was measured in 6 trials. Major changes occurred in all groups of Fritz et al., (2013) $-4.9 \mathrm{~cm} \mathrm{p}<0.001$ (group of normal glucose tolerance), $-2.4 \mathrm{~cm} \mathrm{p}<0.05$ (group of intolerance glucose) and $1.3 \mathrm{~cm} \mathrm{p}<0.05$ (diabetes type 2); Chmelo et al., (2015) obtained significant changes in the aerobic exercise group $-1.8 \mathrm{~cm} p<0.05$ and in resistance training group $-1.5 \mathrm{~cm} p<0.05$; Lunt et al., (2014) obtained change in the intervention group of $-3 \mathrm{~cm} p<0.001$; Heydari et al., (2013) obtained changes of $-3,7 \mathrm{~cm} p<0.05$ and Balducci et al., (2012) $-3.8 p<0.001$. The last variable is the lipid profile. Only 3 of the 10 investigations included the lipid profile among their study variables. In 2 of them, the changes were significant: In Lunt et al., (2014) the total cholesterol decreased by $-7 \mathrm{mg} / \mathrm{dl} p=0.025$ and in Balducci et al., (2012) the LDL cholesterol decreased significantly $-0.41 \mathrm{nmol} p=0.04$.

\section{DISCUSSION}

In this systematic review where we aimed to establish the results in different variables related to the health of interventions based on unsupervised physical activity in sedentary and overweight / obese people and compare them with the results obtained in supervised aerobic trend exercise interventions in the same population were included 17 manuscripts. The 17 study included 9530 subjects, where 3916 were women $(60 \%)$ and 2614 were man (40\%).

The main finding of systematic review was that independently of the intervention applied, improvements were obtained in some variables studied. Previous studies reaffirm the benefits of exercise and physical activity in many of the variables analyzed (Hebden, Chey, \& Allman-Farinelli, 2012; Reed et al., 2014; Ross, Hudson, Stotz, \& Lam, 2015) with both types of interventions, however it is not established what type of intervention is most recommended in each case. For example, Miller et al., (2013) analyzed the results of studies that used exercise to lower the weight of obese people. The results on the variable weight were from -3.6 to -12.6 $\mathrm{kg}$ depending on the test evaluated and observed mayor differences in the variable body fat with results ranging from -6.5 reductions to even $-22 \%$ of body fat. But, it is necessary to carry out a more exhaustive analysis, as well as the protocol and the duration of the studies. There is also the impossibility of isolating the effects of exercise due to the caloric restriction that was included in the intervention and that alters the results.

Ross et al., (2015), analyzed 300 obese subjects who were prescribed exercise. The total group was divided into several groups according to intensity and volume. In all the groups the waist circumference was significantly reduced both at 16 and 24 weeks, as observed in our study $(-3.1$ to $-4.6 \mathrm{~cm})$. 
On the other hand, the results obtained in the weight variable in the unsupervised interventions of our review, are similar to those found by Hebden et al., (2012), where they observed a weight reduction from 0 to $-3.5 \mathrm{~kg}$ after applying an intervention on lifestyle. Nicklas et al., (2014), determined whether adding a self-regulatory intervention focused on self-monitoring of spontaneous physical activity and sedentary behavior to a standard weight loss. To do this, the sample was divided into two groups, one that made diet plus aerobic exercise and the other that was dieting, aerobic exercise and was instructed to improve spontaneous physical activity and decrease sedentary behaviour. After 5 months of intervention, the group that was instructed to reduce sedentary time lost an average of $8.8 \mathrm{~kg}$ compared to the $6.5 \mathrm{~kg}$ of the group, only diet and exercise. In addition, a follow-up of another 5 months was carried out and while the group only diet and exercise recovered $+1.6 \mathrm{~kg}$, the group with spontaneous physical activity almost did not recover, $+0.3 \mathrm{~kg}$. It is observed that the addition of a self-regulatory intervention, designed to increase spontaneous physical activity and decrease sedentary behavior, to a standard weight loss intervention improves the successful maintenance of weight loss. Therefore, here we talk about the term adherence, that is, not about the number of subjects that complete a given intervention, but about the ability of people to continue carrying out the guidelines set out in the study on the amount of physical activity that they have to carry out or on the calories they have to restrict for weight loss. This opens a new line of research to really know what type of intervention greater adherence has, in addition to being effective on variables related to health.

\section{CONCLUSIONS}

Although this systematic review showed similar results in both types of interventions (unsupervised intervention and supervised aerobic exercises intervention), there is not enough evidence to conclude which type of intervention is better. Therefore, further studies on this line of research are necessary to determine if there are benefits of one type of intervention over another and thus be able to recommend with a foundation a typology of physical activity.

\section{REFERENCES}

Carr, L. J., Karvinen, K., Peavler, M., Smith, R., \& Cangelosi, K. (2013). Multicomponent intervention to reduce daily sedentary time: a randomised controlled trial. BMJ Open, 3(10), e003261. https://doi.org/10.1136/bmjopen-2013-003261

Carroll, S., Marshall, P., Ingle, L., \& Borkoles, E. (2012). Cardiorespiratory fitness and heart rate recovery in obese premenopausal women. Scandinavian Journal of Medicine \& Science in Sports, 22(6), e133-e139. https://doi.org/10.1111/j.1600-0838.2012.01522.x

Chmelo, E. A., Crotts, C. I., Newman, J. C., Brinkley, T. E., Lyles, M. F., Leng, X., ... Nicklas, B. J. (2015). Heterogeneity of Physical Function Responses to Exercise Training in Older Adults. Journal of the American Geriatrics Society, 63(3), 462-469. https://doi.org/10.1111/jgs.13322

Dutheil, F., Lac, G., Lesourd, B., Chapier, R., Walther, G., Vinet, A., ... Courteix, D. (2013). Different modalities of exercise to reduce visceral fat mass and cardiovascular risk in metabolic syndrome: the RESOLVE* randomized trial. International Journal of Cardiology, 168(4), 3634-3642. https://doi.org/10.1016/j.ijcard.2013.05.012

Eriksson, M., Uddén, J., Hemmingsson, E., \& Agewall, S. (2010). Impact of physical activity and body composition on heart function and morphology in middle-aged, abdominally obese women. Clinical Physiology and Functional Imaging, 30(5), no-no. https://doi.org/10.1111/j.1475-097X.2010.00952.x

Foster, J. A., Gore, S. A., \& West, D. S. (n.d.). Altering TV viewing habits: an unexplored strategy for adult obesity intervention? American Journal of Health Behavior, 30(1), 3-14. https://doi.org/10.5555/ajhb.2006.30.1.3 
Garber, C. E., Blissmer, B., Deschenes, M. R., Franklin, B. A., Lamonte, M. J., Lee, I.-M., ... American College of Sports Medicine. (2011). Quantity and Quality of Exercise for Developing and Maintaining Cardiorespiratory, Musculoskeletal, and Neuromotor Fitness in Apparently Healthy Adults. Medicine \& Science in Sports \& Exercise, 43(7), 1334-1359. https://doi.org/10.1249/MSS.0b013e318213fefb

Goldfield, G., Saunders, T., P. Kenny, G., Hadjiyannakis, S., Phillips, P., Alberga, A., ... Sigal, R. (2013). Screen Viewing and Diabetes Risk Factors in Overweight and Obese Adolescents. American Journal of Preventive Medicine (Vol. 44). https://doi.org/10.1016/..amepre.2012.11.040

Hebden, L., Chey, T., \& Allman-Farinelli, M. (2012). Lifestyle intervention for preventing weight gain in young adults: a systematic review and meta-analysis of RCTs. Obesity Reviews, 13(8), 692-710. https://doi.org/10.1111/j.1467-789X.2012.00990.x

Heydari, M., Boutcher, Y. N., \& Boutcher, S. H. (2013). The effects of high-intensity intermittent exercise training on cardiovascular response to mental and physical challenge. International Journal of Psychophysiology, 87(2), 141-146. https://doi.org/10.1016/J.IJPSYCHO.2012.11.013

Kempf, K., \& Martin, S. (2013). Autonomous exercise game use improves metabolic control and quality of life in type 2 diabetes patients - a randomized controlled trial. BMC Endocrine Disorders, 13, 57. https://doi.org/10.1186/1472-6823-13-57

Kline, C. E., Crowley, E. P., Ewing, G. B., Burch, J. B., Blair, S. N., Durstine, J. L., ... Youngstedt, S. D. (2011). The Effect of Exercise Training on Obstructive Sleep Apnea and Sleep Quality: A Randomized Controlled Trial. Sleep, 34(12), 1631-1640. https://doi.org/10.5665/sleep.1422

León-Latre, M., Moreno-Franco, B., Andrés-Esteban, E. M., Ledesma, M., Laclaustra, M., Alcalde, V., ... Casasnovas, J. A. (2014). Sedentarismo y su relación con el perfil de riesgo cardiovascular, la resistencia a la insulina y la inflamación. Revista Española de Cardiología, 67(6), 449-455. https://doi.org/10.1016/J.RECESP.2013.10.017

Liberati, A., Altman, D. G., Tetzlaff, J., Mulrow, C., Gøtzsche, P. C., loannidis, J. P. A., .. Moher, D. (2009). The PRISMA statement for reporting systematic reviews and meta-analyses of studies that evaluate healthcare interventions: explanation and elaboration. BMJ, 339. https://doi.org/10.1136/bmi.b2700

Lunt, H., Draper, N., Marshall, H. C., Logan, F. J., Hamlin, M. J., Shearman, J. P., ... Frampton, C. M. A. (2014). High Intensity Interval Training in a Real World Setting: A Randomized Controlled Feasibility Study in Overweight Inactive Adults, Measuring Change in Maximal Oxygen Uptake. PLoS ONE, 9(1), e83256. https://doi.org/10.1371/journal.pone.0083256

Maher, C. G., Sherrington, C., Herbert, R. D., Moseley, A. M., \& Elkins, M. (2003). Reliability of the PEDro scale for rating quality of randomized controlled trials. Physical Therapy, 83(8), 713-21. Retrieved from http://www.ncbi.nlm.nih.gov/pubmed/12882612

Martínez-Gómez, D., Eisenmann, J. C., Gómez-Martínez, S., Veses, A., Marcos, A., \& Veiga, O. L. (2010). Sedentarismo, adiposidad y factores de riesgo cardiovascular en adolescentes. Estudio AFINOS. Revista Española de Cardiología, 63(3), 277-285. https://doi.org/10.1016/S03008932(10)70086-5

Miller, C. T., Fraser, S. F., Levinger, I., Straznicky, N. E., Dixon, J. B., Reynolds, J., \& Selig, S. E. (2013). The Effects of Exercise Training in Addition to Energy Restriction on Functional Capacities and Body Composition in Obese Adults during Weight Loss: A Systematic Review. PLoS ONE, 8(11), e81692. https://doi.org/10.1371/journal.pone.0081692

Nicklas, B. J., Gaukstern, J. E., Beavers, K. M., Newman, J. C., Leng, X., \& Rejeski, W. J. (2014). Selfmonitoring of spontaneous physical activity and sedentary behavior to prevent weight regain in older adults. Obesity, 22(6), 1406-1412. https://doi.org/10.1002/oby.20732

Owen, N. (2012). Sedentary behavior: Understanding and influencing adults' prolonged sitting time. Preventive Medicine, 55(6), 535-539. https://doi.org/10.1016/j.ypmed.2012.08.024 
Pate, R. R., O'Neill, J. R., \& Lobelo, F. (2008). The Evolving Definition of \&quot;Sedentary\&quot; Exercise and Sport Sciences Reviews, 36(4), 173-178. https://doi.org/10.1097/JES.0b013e3181877d1a

Peeters, G., Mishra, G., Dobson, A., \& Brown, W. (2014). Health Care Costs Associated with Prolonged Sitting and Inactivity. American journal of preventive medicine (Vol. 46). https://doi.org/10.1016/i.amepre.2013.11.014

Reed, J. L., Prince, S. A., Cole, C. A., Fodor, J. G., Hiremath, S., Mullen, K.-A., ... Reid, R. D. (2014). Workplace physical activity interventions and moderate-to-vigorous intensity physical activity levels among working-age women: a systematic review protocol. Systematic Reviews, 3, 147. https://doi.org/10.1186/2046-4053-3-147

Rejeski, W. J., Ip, E. H., Bertoni, A. G., Bray, G. A., Evans, G., Gregg, E. W., \& Zhang, Q. (2012). Lifestyle Change and Mobility in Obese Adults with Type 2 Diabetes. New England Journal of Medicine, 366(13), 1209-1217. https://doi.org/10.1056/NEJMoa1110294

Rodríguez-Hernández, A., Molina, S. F., Martínez-Santos, R., \& Cruz-Sánchez, E. de la. (2011). Prevalencia Y Distribución De La Inactividad Física Y El Exceso De Peso En La Población Española En Edad Escolar. E-Balonmano.Com: Revista de Ciencias Del Deporte, 7(3), 157-168. Retrieved from http://www.e-balonmano.com/ojs/index.php/revista/article/view/97

Rosenkilde, M., Reichkendler, M. H., Auerbach, P., Toräng, S., Gram, A. S., Ploug, T., ... Stallknecht, B. (2013). Appetite regulation in overweight, sedentary men after different amounts of endurance exercise: a randomized controlled trial. Journal of Applied Physiology, 115(11), 1599-1609. https://doi.org/10.1152/japplphysiol.00680.2013

Ross, R., Hudson, R., Stotz, P. J., \& Lam, M. (2015). Effects of Exercise Amount and Intensity on Abdominal Obesity and Glucose Tolerance in Obese Adults. Annals of Internal Medicine, 162(5), 325. https://doi.org/10.7326/M14-1189

Steeves, J. A., Bassett, D. R., Fitzhugh, E. C., Raynor, H. A., \& Thompson, D. L. (2012). Can sedentary behavior be made more active? A randomized pilot study of TV commercial stepping versus walking. International Journal of Behavioral Nutrition and Physical Activity, 9(1), 95. https://doi.org/10.1186/1479-5868-9-95

Thorp, A. A., Owen, N., Neuhaus, M., \& Dunstan, D. W. (2011). Sedentary Behaviors and Subsequent Health Outcomes in Adults. American Journal of Preventive Medicine, 41(2), 207-215. https://doi.org/10.1016/j.amepre.2011.05.004

Tremblay, M. S., Aubert, S., Barnes, J. D., Saunders, T. J., Carson, V., Latimer-Cheung, A. E., ...Chinapaw, M. J. M. (2017). Sedentary Behavior Research Network (SBRN) - Terminology Consensus Project process and outcome. International Journal of Behavioral Nutrition and Physical Activity, 14(1), 75. https://doi.org/10.1186/s12966-017-0525-8

Tremblay, M. S., Colley, R. C., Saunders, T. J., Healy, G. N., \& Owen, N. (2010). Physiological and health implications of a sedentary lifestyle. Applied Physiology, Nutrition, and Metabolism, 35(6), 725-740. https://doi.org/10.1139/H10-079

van der Ploeg, H. P., Chey, T., Korda, R. J., Banks, E., \& Bauman, A. (2012). Sitting Time and All-Cause Mortality Risk in 222497 Australian Adults. Archives of Internal Medicine, 172(6), 494. https://doi.org/10.1001/archinternmed.2011.2174

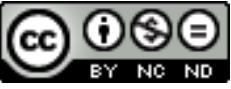

This title is licensed under a Creative Commons Attribution-NonCommercial-NoDerivs 4.0 Unported License. 